\title{
DYNAMIC ALLIANCE OF AGRICULTURE PRODUCTS LOGISTICS BASED ON SWARM INTELLIGENCE
}

\author{
Xinsheng Yao ${ }^{*}$, Yan Cui, Jilai Ying, Jianguang Wei \\ College of Mechanical and Electrical Engineering, Henan Agricultural University, \\ Zhengzhou, Henan Province, P. R. China 450002 \\ * Corresponding author, Address: College of Mechanical and Electrical Engineering, Henan \\ Agricultural University, Zhengzhou, 450002, Henan Province, P. R. China, Tel: +86-371- \\ 63978179; FAX:+86-371-63558368; Email: yaoxsh@163.com
}

\begin{abstract}
Along with the growing up of the Chinese generalized agriculture, the agriculture products logistics demands are increasing quickly in quality and quantity. Oppositely, the service of agriculture products logistics is slowly. It is very essential to study the logistics service mode suited to the tendency of the agriculture products logistics demand. The paper analyzes the common characteristic between the agriculture products logistics individual and the intelligence individual. Then, by the swarm intelligence, the dynamic alliance of agriculture products logistics is presented, the construction algorithm and the application method are given too. The paper provides a better operable development mode for the agriculture products logistics in China, which has directive meaning to improve the logistics efficiency for the socialistic new economy development and the New County Construction.
\end{abstract}

Keywords: agriculture products logistics, swarm Intelligence; dynamic alliance; multi individual

\section{INTRODUCTION}

Benefited from the Three Agricultural Policies and New Country Construction, Chinese generalized agriculture is growing up, agriculture and related industries are developing quickly. And the demand of agriculture

Please use the following format when citing this chapter:

Yao, X., Cui, Y., Ying, J. and Wei, J., 2009, in IFIP International Federation for Information Processing, Volume 293, Computer and Computing Technologies in Agriculture II, Volume 1, eds. D. Li, Z. Chunjiang, (Boston: Springer), pp. 761-769. 
products logistics is also increasing quickly in quality and quantity. Influenced by the thought 'emphasized production and despised circulation' in the traditional agriculture economy in China, agriculture products logistics in China started late and develops slowly, characterized by small-scale peasant economy; which limits the agriculture development and the production, circulation of the agriculture products. Thus, it is very essential to study the service mode of the logistics suited to the tendency to the agriculture products logistics demand.

Now, there are various problems in the agriculture products logistics in China, including low efficiency, poor quality, low informatization, few manpower etc.. For logistics individual, there are various modes, including the large enterprise; medium and small enterprise, self-employed individuals and farmers. Between these individuals, there are great difference in operation, management, specialty, lacking organization and platform. For the particularity of the agriculture products and its logistics, these are little logistics enterprise which can operate all kind agriculture products logistics. In fact, many logistics individual are competing repeatedly in low efficiency, low level market. The whole efficiency is very low. For instance, the proportion of the loss of vegetable product in logistics is about $5 \%$ in developed countries, $1-2 \%$ in America, but as high as $25-30 \%$ in China. That is, almost 80 millions tons vegetable is wasted every year (Zhao, 2005). If agricultural products logistics cost can be reduced, so is agricultural production expense, which leads to the increase of farmers' income.

For the rapid developing of the agriculture products logistics market and the problem of the logistics individual, it is essential to integrate the agriculture logistics service. The integrated object includes farmers, former material supplier, agriculture products collector, packing, transportation, warehousing, marketing, product planning, and information service (Meng, 2005). However, the integration on entity is very difficult, for a lot unfathomed problem in the operation management, benefit distribution etc.. And it is impossible to exist long-term stable organization for there is much difference between the individuals in the domain, manpower, ability and idea etc.. Then, the dynamic logistic organization has more advantage. At present, some research about logistics integration is mainly for industry logistics (Dai, 2004), little on agriculture products logistics.

The paper reveals that the agriculture products individual have the characteristic similar to the intelligence individual. By the swarm intelligence, the dynamic alliance of agriculture products logistics is presented, the construction algorithm for the alliance and the management of the alliance members are analyzed. Then, the creating, running, dissolving of the agriculture products logistics dynamic alliance is discussed. 
Intelligence

\section{SWARM INTELLIGENCE AND AGRICULTURE PRODUCTS LOGISTICS ALLIANCE}

Agriculture products logistics individual, has a familiar cognition with itself, can make one or some logistics operation, which is looked as an individual with some level intelligence. By swarm intelligence, some agriculture products logistics may build up a dynamic alliance responding to the coming logistic job.

\subsection{Intelligence individual and agriculture products logistics individual}

The definition and explain of the intelligence individual have many edition. The intelligence mainly appears as some form as software or hardware of computer. Yet, they all have some commonness, such as, the highly adaptability and autonomy. Shoham (Shoham, 1993) regards that an entity is intelligence individual if its state includes the psychosis, such as knowledge, idea, promise and ability etc..

An individual which implements some operation of agriculture products logistics, as an independent entity which has knowledge, idea, ability and social duty, can be looked as intelligence individual. Then, the agriculture products logistics individual, as an intelligence individual, has the commonness: autonomy, social ability, flexibility, mobility, reaction, consciousness. This is the foundation for the making for the swarm intelligence alliance operation.

\subsection{Multi individual swarm intelligence}

In general, swarm intelligence is the characteristic which the entity without intelligence behaved intelligently. Swarm intelligence provides an approach to solve the distributed problem, but no need of centralized control and global model. By applying swarm intelligence, some algorithms have the characteristic of intelligence. By the interaction between the individual and environment, the algorithm has some self-organization function, robustly and visually. And, the theory of multi individual system is from distributed artificial intelligence, applied in many domains widely. Multi individual system mainly studies the corresponded intelligence action of the multi individual separated in logic or physics, and at last solves the problem.

Swarm intelligence based on multi individual, not only exerts the initiative, independence of the individual, but also makes the colony has the additional 
function than the simple combined members have, presents more intelligence functions than normal intelligence individual.

\subsection{Agriculture products logistics dynamic alliance}

Under the well cooperation mechanism and fellowship, agriculture products logistics individuals connect and cooperate in related logistics work, then they can buildup logistics alliance (Bouckova, 2002). In fact, the logistics alliance is an integration platform based on information (Gunasekaran, 2004). Agriculture products logistics alliance includes the function like a real logistics enterprise; can implement the same operation, like warehousing, transportation, packing and etc..

In the environment of agriculture products logistics integration, the relation between the individuals is the cooperation fellowship based on credit, cooperation, and 'win-win'. Like the traditional integration mode, the fixed logistics alliance has many solidified default, e.g., the low adaptability for operation. Then the alliance needs to create and dissolve usually to adapt the logistics demand, which will waste more manpower, capital and resource, reduce the efficiency, and affect the fellowship between the individuals.

By the multi individual swarm intelligence, the paper abstracts the fellowship in the alliance construction, then buildup the agriculture logistics dynamic alliance adapted to the agriculture products logistics. Based on the dynamic alliance mechanism, every member understands the participant logistics operation deeply. Then, in cooperation, the members can implement the logistics work which can not by itself. The dynamic organization is more helpful to respond to the market rapidly, can exert more superiority and scale merit than the large enterprise.

\section{THE MECHANISM OF THE AGRICULTURE PRODUCTS LOGISTICS DYNAMIC ALLIANCE}

Agriculture products logistics dynamic alliance is timely and dynamic, creating for the logistics task and dissolving as the task end. The main idea of the formation algorithm of the dynamic alliance is as follows. At first, the system is droved by the coming logistics task; then the task is analyzed and decomposed. According to the sub task, the first generation combination of the logistics individual is generated randomly. Where, each sub task can include one or several logistics individuals. Then, each selected individual can select a group cooperation team with good fellowship. Repeating it, until the combination is stable, this is the last combination the task need. 


\subsection{Agriculture products logistics individuals database management}

Based on the understanding about the agriculture products logistics, the agriculture products logistics individual member database is formed. The database keeps the information of the logistics members, including operation service domain, operation type, cooperator, enterprise scale, operation comprehensive assessment etc.. System formats the candidate logistics individual and adds it into the database. System can also maintain and make other operation to the member. Logistics individual member database and its management showed in Fig. 1.

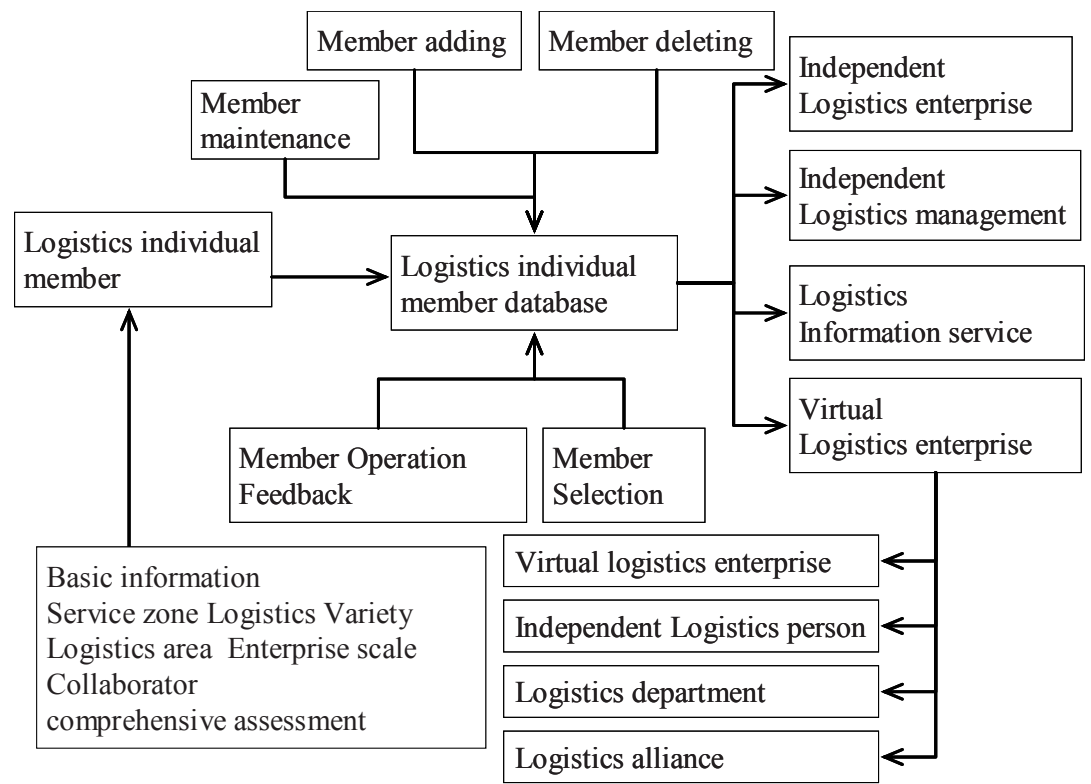

Fig.5. Agriculture products logistics individual member management in the dynamic alliance

There, the operation service range include: pre-logistics of agriculture products, primary gathering, primary processing, and warehousing, packing, transportation, distributing and information service. There includes two type basic individual, one is the entity logistics enterprise, which is the same as it in the real world; the other is virtual logistics enterprise, which maybe a department of a logistics enterprise, or a virtual enterprise combined in some approach. For some large agriculture products logistics enterprise, it can be added in the database as an independent enterprise, or can be decomposed as several virtual logistics operation and then added in database. Apparently, these virtual logistics enterprise decomposed by a large enterprise have more 
near relationship, may select each other when cooperation. But in other environment, for efficiency and other reason, some integrated enterprise will select other fellow to complete a logistics work though it has sub enterprise about that work.

\subsection{The algorithm of agriculture products logistics dynamic alliance}

For a given logistics task, a multidimensional index space is formed, including the time, place, equipment, enterprise ability and service quality of the logistics task. The same number intelligence individuals are placed in the multidimensional space randomly, where each intelligence individual corresponding to a logistics sub task. The intelligence individuals are moving in the space and evaluated. At last, they are integrated by the multidimensional indexes.

Assume that the logistics member data set is $O=\left\{O_{i}=\left(o_{i 1}, o_{i 2}, \ldots, o_{i m}\right)\right.$, $i=1,2, \ldots, N\}$, is a set including $N$ agricultural products logistics enterprise members. For one logistics intelligence individual $O_{i}, o_{i 1}, o_{i 2}, \ldots, o_{i m}$ are the performance index described before. Let

$$
d_{i j}=\left\|P\left(O_{i}-O_{j}\right)\right\|=\sqrt{\sum_{k=1}^{m} w_{k}\left(o_{i k}-o_{j k}\right)^{2}}
$$

Where $d_{i j}\left(O_{i}, O_{j}\right)$ is the Euclidean distances between $O_{i}$ and $O_{j}$; expresses the cooperation willing between $O_{i}$ and $O_{j}$ in real world, $P$ expresses the weighted integration, $w_{k}$ is the weight, determined by the importance degree of the performance to a logistics task.

Before the selection of the individual cooperation, a candidate $\operatorname{area}\left(O_{i}, d_{r}\right)$ are given, as the cooperation field of data object $O_{i}$, which expresses a group logistics individuals which cooperate nearly. $d_{r}$ is the cooperation willing radius, expresses an integrated value of indexes.

When selection, $O_{i}$ selects a cooperation group $A=\left\{A_{i}=\left(a_{i 1}, a_{i 2}, \ldots, a_{i k}\right)\right.$, $\left.a_{i k} \in O\right\}$ in $\operatorname{area}\left(O_{i}, d_{r}\right)$, which satisfied the sub task. The selection basis of each $a_{i k}$ is its $d_{i j}$ is the least in area $a\left(O_{i}, d_{r}\right)$.

$$
d_{i j}\left(a_{i k}\right) \leq d_{i j}\left(a_{i l}\right), a_{i l} \in \text { area }\left(O_{i}, d_{r}\right)
$$

Based on the group selection algorithm, the algorithm for the agriculture products logistics dynamic alliance is as follows.

(1) Task analysis and decomposing. The logistics task from the management system is analyzed and decomposed by the type described in Fig.1. The sub task type $\mathrm{m}$ and task require (sub task) $\mathrm{Q}=\{\mathrm{q} 1, \mathrm{q} 2, \ldots$, $\mathrm{qm}\}$ are generated. The object evaluated index $\mathrm{B}=\{\mathrm{b} 1, \mathrm{~b} 2, \ldots, \mathrm{bm}\}$ are generated at the same time. Set the round number e. 
(2) Alliance initialization. By the sub task Q, m intelligence individuals are generated from the database randomly, and the parameters (efficiency, domain, time and other) corresponding $\mathrm{B}$ are generated at the same time. This part makes randomly, or can be done by one task founder. These $\mathrm{m}$ intelligence individuals compose the first generation group.

(3) Generate the criterion for the intelligence individual to select cooperator. The first is the characteristic of the task (time domain, space domain, task composing, task volume, task precision, industry scope, etc.), then is cooperation relationship, business system etc.. Considering these factors synthetically, an intelligence individual corresponding to a sub logistics task, can select one or several cooperators. After a round selection for all intelligence individual in the last round group, a next generation group generates and replaces the old one. And select the new dr. This step mainly adopts the algorithm described before.

(4) For $\mathrm{i}=1,2, \ldots, \mathrm{n}$, for each intelligence individual in the group, observe radius $\mathrm{dr}$, search optimal cooperator group. In search, in the area(Oi, dr), by the need of the sub task, calculate the cooperator distance dij, if little than the individual in the candidate group, replaced the old one with it.

(5) After a round search, an alliance scheme generated. For the individuals in the alliance are from different selection of each individual in the last round, the individual in the alliance scheme may conflict each other, and then there need a filter. The criterion for filter is that in an alliance, there only keep the optimal individual group to complete the sub task. The filter algorithm can adopt many modes. The simple mode 1 is to limit the capability of each logistics sub work for the task. The simple mode 2 is to limit the individual counts in each round group.

(6) Judge if the algorithm should end. If end, goto step 7; or goto step 4 to start a new round to generate a new alliance scheme. The end condition can be the search times arrives the round number e. Or the condition is the object index arrive the precision. i.e., the algorithm has convergent.

(7) Assign the task to the real enterprise and implement it.

(8) After the task completes, by the performance of the member in this task, adjust the evaluation of the logistics individual member and give feedback to the database.

\section{THE IMPLEMENT OF THE AGRICULTURE PRODUCTS LOGISTICS DYNAMIC ALLIANCE}

Agriculture products logistics dynamic alliance, cored by the cooperation relation selection algorithm based on the multi individual swarm, droved by 
logistics task, sustain by logistics individual database management system, implementing the integration of the agriculture products logistics. The main structure is shown in Fig. 2.

The operation of the agriculture products dynamic alliance includes the creating, running and dissolving. Each member implements one logistics operation, and with the cooperation the alliance completes the logistics task in the end.

In the course of implement, the management system and information platform provide the connection for the logistics individuals. There, each logistics individual member can accept order. Then, based on this algorithm, by the share information platform and the logistics individual database this individual can acquire the necessary information, select the cooperator and form the dynamic alliance, then to carry out the logistics job.

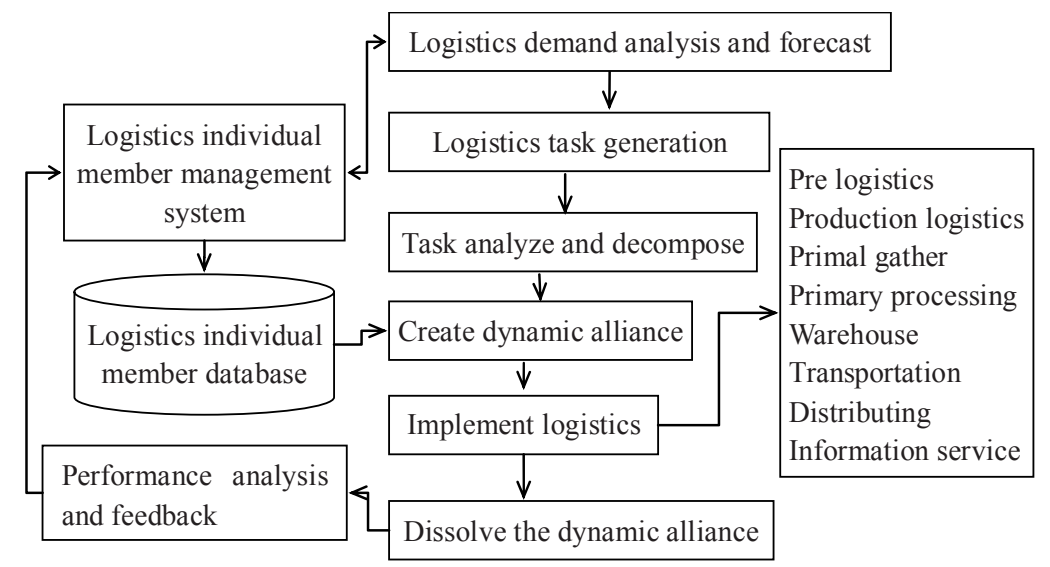

Fig.6. The implement of the agriculture products logistics dynamic alliance

\section{CONCLUSION}

There exist serious dissymmetry between the quick increasing demand of agriculture products logistics and the slow service. Agriculture products logistics dynamic alliance provided a suitable mode for agriculture products logistics. The paper presents a member cooperation fellow selection algorithm based on multi individual swarm intelligence. Based on this algorithm, little and media logistics individual can organize together dynamically. One side, this method can exert the advantage and improve the efficiency of the member; the other, the service ability and quality can be improved. Based on the algorithm, the paper studies the creating, running, dissolving of the agriculture products logistics dynamic alliance, which theory is feasible. Because the application need great data, the algorithm just 
has been emulating in lab. In emulation, among the given 30 logistics enterprises, a vegetable logistics dynamic alliance composed by 7 enterprises have generated, which performance excels than a actual selection by a group of experts. At last, in application, there are many problem need solve, including the alliance management, credit system, performance evaluation etc.. The depth on theory and width of application are the tendency of the following study.

\section{REFERENCES}

Bouckova, B. Agricultural co-operatives: perspectives for the 21st century, Journal of Agricultural Economics, 2002, 48(4): 166-170

Dai, Y., Lu J.Q.: Internet-based Virtual Logistics Business Alliance IT Platform. LOGISTICS SCI TECH, 2001,5: 11-13 (in Chinese)

Gunasekaran, A., Ngai, E.: Information systems in supply chain integration and management, European Journal of Operational Research, 2004 159: 269-295

Meng, F. Sh., Jin, M. H.: The Research on the Reason of Logistics of Agricultural Products Lagging LOGISTICS SCI TECH, 2005,28(3): 67-69 (in Chinese)

Opara, L.: Traceability in Agriculture and Food Supply Chain Food. Agriculture \& Environment, 2003,1(1): 101-106

Shoham Y. Agent-oriented programming. Artificial Intelligence, 1993, 51-92

Zhao, C.J.: Logistics Existing Problem and Counter measure of Agricultural Produce. Journal Of Tianjin University of Commerce, 2005,25(4): 41-44(in Chinese) 